\title{
Penile Carcinoma In Situ
}

National Cancer Institute

\section{Source}

National Cancer Institute. Penile Carcinoma In Situ. NCI Thesaurus. Code C27790.

Stage 0 includes: Tis, NO, MO. T is: Carcinoma in situ. cN0: No palpable or visibly enlarged inguinal lymph nodes. pNO: No regional lymph node metastasis. MO: No distant metastasis. (AJCC 7th ed.) 